\title{
A young bipolar outflow from IRAS 15398-3359
}

\author{
P. Bjerkeli ${ }^{1,2}$, J. K. Jørgensen ${ }^{1}$, and C. Brinch ${ }^{3}$
}

\author{
${ }^{1}$ Centre for Star and Planet Formation, Niels Bohr Institute \& Natural History Museum of Denmark, University of Copenhagen, \\ Øster Voldgade 5-7, 1350 Copenhagen K., Denmark \\ e-mail: per.bjerkeli@nbi.dk \\ 2 Department of Earth and Space Sciences, Chalmers University of Technology, Onsala Space Observatory, 43992 Onsala, Sweden \\ 3 Niels Bohr International Academy, The Niels Bohr Institute, Blegdamsvej 17, 2100 Copenhagen, Denmark
}

Received 4 September 2015 / Accepted 14 January 2016

\section{ABSTRACT}

\begin{abstract}
Context. Changing physical conditions in the vicinity of protostars allow for a rich and interesting chemistry to occur. Heating and cooling of the gas allows molecules to be released from and frozen out on dust grains. These changes in physics, traced by chemistry as well as the kinematical information, allows us to distinguish between different scenarios describing the infall of matter and the launching of molecular outflows and jets.

Aims. We aim to determine the spatial distribution of different species that are of different chemical origin. This is to examine the physical processes in play in the observed region. From the kinematical information of the emission lines we aim to determine the nature of the infalling and outflowing gas in the system. We also aim to determine the physical properties of the outflow.

Methods. Maps from the Submillimeter Array (SMA) reveal the spatial distribution of the gaseous emission towards IRAS 15398-3359. The line radiative transfer code LIME is used to construct a full 3D model of the system taking all relevant components and scales into account.

Results. $\mathrm{CO}, \mathrm{HCO}^{+}$, and $\mathrm{N}_{2} \mathrm{H}^{+}$are detected and shown to trace the motions of the outflow. For $\mathrm{CO}$, the circumstellar envelope and the surrounding cloud also have a profound impact on the observed line profiles. $\mathrm{N}_{2} \mathrm{H}^{+}$is detected in the outflow, but is suppressed towards the central region, perhaps because of the competing reaction between $\mathrm{CO}$ and $\mathrm{H}_{3}^{+}$in the densest regions as well as the destruction of $\mathrm{N}_{2} \mathrm{H}^{+}$by CO. $\mathrm{N}_{2} \mathrm{D}^{+}$is detected in a ridge south-west of the protostellar condensation and is not associated with the outflow. The morphology and kinematics of the $\mathrm{CO}$ emission suggests that the source is younger than 1000 years. The mass, momentum, momentum rate, mechanical luminosity, kinetic energy, and mass-loss rate are also all estimated to be low. A full 3D radiative transfer model of the system can explain all the kinematical and morphological features in the system.
\end{abstract}

Key words. ISM: individual objects: IRAS 15398-3359 - ISM: molecules - ISM: abundances - ISM: jets and outflows stars: winds, outflows

\section{Introduction}

When stars form, several distinctly different physical components are present in the region, i.e. a protoplanetary disk, a collapsing protostellar envelope, and a (bipolar) molecular outflow. The chemistry in these regions is complex and the abundance of various species varies with the changing physical conditions in time and space. Heating from the protostar and shocks can evaporate molecules into the gas-phase, but molecules can also freeze out in the regions where temperatures are low.

The Class 0 (André et al. 1990, 1993) protostar IRAS 15398-3359 (Shirley et al. 2000) is located in the Lupus I cloud $\left(\alpha_{2000}=+15^{\mathrm{h}} 43^{\mathrm{m}} 02^{\mathrm{s}} 2 ; \delta_{2000}=-34^{\circ} 09^{\prime} 06.7^{\prime \prime} ;\right.$ Jørgensen et al. 2013) at a distance of $155 \mathrm{pc}$ (Lombardi et al. 2008). The source has a bolometric temperature of $44 \mathrm{~K}$ (Jørgensen et al. 2013) and is known to harbour a molecular outflow (e.g. Tachihara et al. 1996; van Kempen et al. 2009b). The region has, however, not attracted much interest until very recently, not least through the ALMA, Cycle 0 observations that were carried out towards this region (Jørgensen et al. 2013; Oya et al. 2014). These observations shows that the source most likely underwent a burst in accretion during the last $100-1000$ years, which was manifested by an absence of $\mathrm{HCO}^{+}$in the vicinity of the protostellar object. IRAS $15398-3359$ is also known to show interesting chemical signatures. Sakai et al. (2011) reported an increase in carbon-chain molecules in the inner regions closest to the protostellar source (500-1000 AU), and this YSO is one of the so-called warm carbon-chain chemistry sources. Since it is one of the very nearby, young outflow sources with an interesting chemistry, this YSO makes an excellent target for detailed studies of the gas morphology and kinematics of different species.

The mass loss, traced by the outflow likely has its origin close to the protostellar object itself (see e.g. Banerjee \& Pudritz 2006; Machida et al. 2008) and it is in fact one of the most spectacular features of the star formation process. The gas is likely ejected through magneto-centrifugal processes (see e.g. Shang et al. 2007; Pudritz et al. 2007), however, the details of these mechanisms are not yet fully understood. Accretion shocks are also believed to be present in the inner region, but at present it is difficult to unambiguously determine if infall occurs in any specific object. Self-absorbed, optically thick spectral lines, where the red-shifted component is enhanced with respect to the blueshifted component, can be a sign of this. However, to distinguish between the different processes, one needs to calculate the radiative transfer, taking all different components into account simultaneously. This clearly calls for full 3D radiative transfer modelling, which has to this date not been carried out. However, studies of YSOs at high spatial and spectral resolutions are necessary to compare such models with observations.

The aim of this paper is to understand the observed kinematical and morphological information in relation to star formation 
Table 1. Correlator set-ups.

\begin{tabular}{|c|c|c|c|c|c|c|c|}
\hline Chunk & Number of channels/resolution & LSB frequency & Line & $E_{\text {up }}(\mathrm{K})$ & USB frequency & Line & $E_{\text {up }}(\mathrm{K})$ \\
\hline \multicolumn{8}{|c|}{$230 \mathrm{GHz}$ dataset (219.448-221.430 and 229.447-231.429 GHz): } \\
\hline 13 & $512 / 0.203 \mathrm{MHz}$ & $220.343-220.447 \mathrm{GHz}$ & ${ }^{13} \mathrm{CO}(2-1)$ & 15.9 & $230.430-230.534 \mathrm{GHz}$ & ${ }^{12} \mathrm{CO}(2-1)$ & 16.6 \\
\hline 14 & $256 / 0.406 \mathrm{MHz}$ & $220.261-220.364 \mathrm{GHz}$ & - & - & $230.513-230.616 \mathrm{GHz}$ & ${ }^{12} \mathrm{CO}(2-1)$ & 16.6 \\
\hline 23 & $512 / 0.203 \mathrm{MHz}$ & $219.529-219.633 \mathrm{GHz}$ & $\mathrm{C}^{18} \mathrm{O}(2-1)$ & 15.8 & $231.244-231.338 \mathrm{GHz}$ & $\mathrm{N}_{2} \mathrm{D}^{+}(3-2)$ & 22.2 \\
\hline \multicolumn{8}{|c|}{$267 \mathrm{GHz}$ dataset (267.499-269.522 and 277.559-279.562 GHz): } \\
\hline 24 & $512 / 0.203 \mathrm{MHz}$ & $267.499-267.603 \mathrm{GHz}$ & $\mathrm{HCO}^{+}(3-2)$ & 25.7 & $279.458-279.562 \mathrm{GHz}$ & $\mathrm{N}_{2} \mathrm{H}^{+}(3-2)$ & 26.8 \\
\hline
\end{tabular}

theory. The outline of the paper is as follows. The observations are described in detail in Sect. 2, and the kinematical and morphological distribution of the detected species are discussed in Sect. 3. In Sect. 4 we analyse the observed line profiles and present a radiative transfer model of the system, taking all different components into account. The physical properties of the source are discussed in Sect. 5. The main conclusions of the paper are outlined in Sect. 6.

\section{Observations}

The data for IRAS 15398-3359 were obtained from the archive of the Submillimeter Array (SMA; Ho et al. 2004). Parts of these data have previously been utilised in papers by Chen et al. (2013) and Jørgensen et al. (2015). The data originate from observations on two occasions: 2009 April 29 and 2009 May 12, covering spectral set-ups at around 230 and $267 \mathrm{GHz}$, respectively. During both observations the array was in its compact configuration covering baselines from about 5-87 k $\lambda(230 \mathrm{GHz})$ and $8-109 \mathrm{k} \lambda(267 \mathrm{GHz})$ resulting in beam sizes of $2.4-4.5^{\prime \prime}$. The observations are sensitive to emission originating on scales smaller than $\sim 20^{\prime \prime}$ (Wilner \& Welch 1994). The phase centre of the observations was $\alpha_{2000}=+15^{\mathrm{h}} 43^{\mathrm{m}} 02^{\mathrm{s}} .16, \delta_{2000}=-34^{\circ} 09^{\prime} 09.0^{\prime \prime}$ in both cases. The SMA correlator was in both instances set to provide a uniform coverage of $0.812 \mathrm{MHz}\left(0.9-1.1 \mathrm{~km} \mathrm{~s}^{-1}\right)$ in the $24104 \mathrm{MHz}$ chunks distributed over each of its upper and lower sidebands, except in some of the chunks covering specific lines where a higher spectral resolution $(0.203-0.406 \mathrm{MHz}$; $0.22-0.56 \mathrm{kms}^{-1}$ ) was chosen (Table 1). For the analysis presented in this paper, as well as the analysis of Jørgensen et al. (2015) focusing solely on the $\mathrm{C}^{18} \mathrm{O}(2-1)$ emission, the data were downloaded from the archive, calibrated using the standard recipes via the IDL/MIR software ${ }^{1}$, and imaged with Miriad (Sault et al. 1995).

In addition to the Submillimeter Array data, we also utilise ALMA Cycle 0 observations of $\mathrm{C}_{2} \mathrm{H}$ from Early Science programme 2011.0.00628.S, which was also previously presented by Jørgensen et al. (2013). Those observations provide maps of the $\mathrm{C}_{2} \mathrm{H} N=4-3$ emission at $349.4 \mathrm{GHz}$ with an angular resolution of approximately $0.5^{\prime \prime}$. For further details about the ALMA data we refer to that paper.

\section{Results}

\subsection{Kinematics}

The ${ }^{12} \mathrm{CO}$ emission emanating from IRAS $15398-3359$ was detected at a high signal-to-noise ratio. The observed ${ }^{12} \mathrm{CO}$ line profiles show a triangular shape with a central absorption at the systemic velocity, $v_{\text {sys }}=+5.5 \mathrm{~km} \mathrm{~s}^{-1}$ (determined by fitting

\footnotetext{
https://www.cfa.harvard.edu/ cqi/mircook.html
}

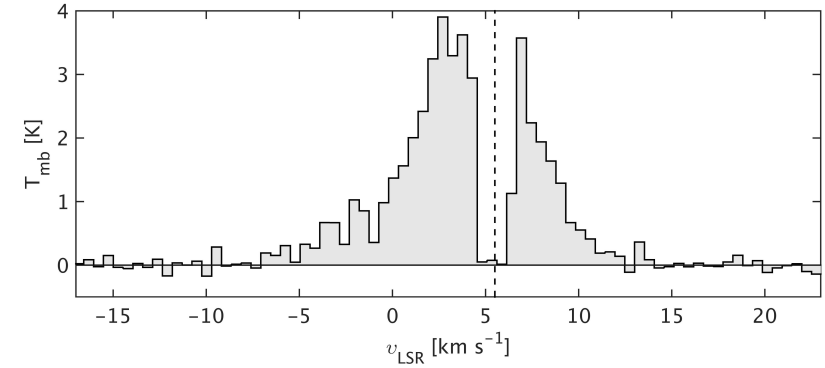

Fig. 1. ${ }^{12} \mathrm{CO}(2-1)$ spectrum towards the central position. The velocity of the source, $v_{\mathrm{LSR}}=+5.5 \mathrm{~km} \mathrm{~s}^{-1}$, is indicated with a dashed vertical line.

Gaussians to the observed spectra), indicative of infall and/or outflow emission. This spectrum is presented in Fig. 1, where the velocity is with respect to $v_{\mathrm{LSR}}$. From here on and throughout this paper, however, all velocities are reported with respect to $v-v_{\text {sys }}$ for clarity. In the other CO isotopologues, and also in several of the other observed species, strong outflow activity and clear blue- and red-shifted asymmetries are observed. The velocity integrated spatial distributions of the detected lines are shown in Fig. 2. Compared to the $1-0$ transition of $\mathrm{CO}$, the 2-1 transition is particularly well suited for kinematical studies (see Sect. 5) because of its higher upper state energy $\left(E_{\mathrm{up}}=16.6 \mathrm{~K}\right)$, which makes it less sensitive to the low-temperature quiescent gas. High-velocity emission (up to $\sim 8 \mathrm{~km} \mathrm{~s}^{-1}$ offset from the systemic velocity) is detected both in the outflow and towards the central source. In Fig. 3, we present a channel map of the region in ${ }^{12} \mathrm{CO}(2-1)$. This figure shows the velocity integrated emission in velocity intervals of $2 \mathrm{~km} \mathrm{~s}^{-1}$. The two upper panels show the gas moving at higher velocities compared to the systemic velocity $\left(\Delta v>5 \mathrm{~km} \mathrm{~s}^{-1}\right)$ while the gas at lower velocities $\left(\Delta v<5 \mathrm{~km} \mathrm{~s}^{-1}\right)$ is presented in the lower panels. In Fig. 4, a position velocity cut along the direction of the outflow $\left(\mathrm{PA}=35^{\circ}\right)$ and the presumed disk-like structure (Oya et al. 2014) is presented. Other species are only detected at a low velocity within $\Delta v \simeq 3 \mathrm{~km} \mathrm{~s}^{-1}$ from systemic velocity. The mass loss is also clearly detected in the ${ }^{13} \mathrm{CO}$ maps, whilst the $\mathrm{C}^{18} \mathrm{O}$ emission is only detected at blue-shifted velocities, closest to the central source. $\mathrm{HCO}^{+}$is detected in the outflow as well, at relatively low radial velocities $\left(\Delta v \sim 3 \mathrm{~km} \mathrm{~s}^{-1}\right)$ compared to $v_{\text {sys }}$ and both red-shifted and blue-shifted emission is detected in both outflow lobes. $\mathrm{N}_{2} \mathrm{H}^{+}$is detected in two knots on each side of the protostar and the lines are narrow, i.e. a few $\mathrm{km} \mathrm{s}^{-1}$. The knots are, however, clearly separated in velocity.

\subsection{Morphology}

The ${ }^{12} \mathrm{CO}$ emission is confined to the two outflow lobes emanating from IRAS 15398-3359, and the morphology of the 


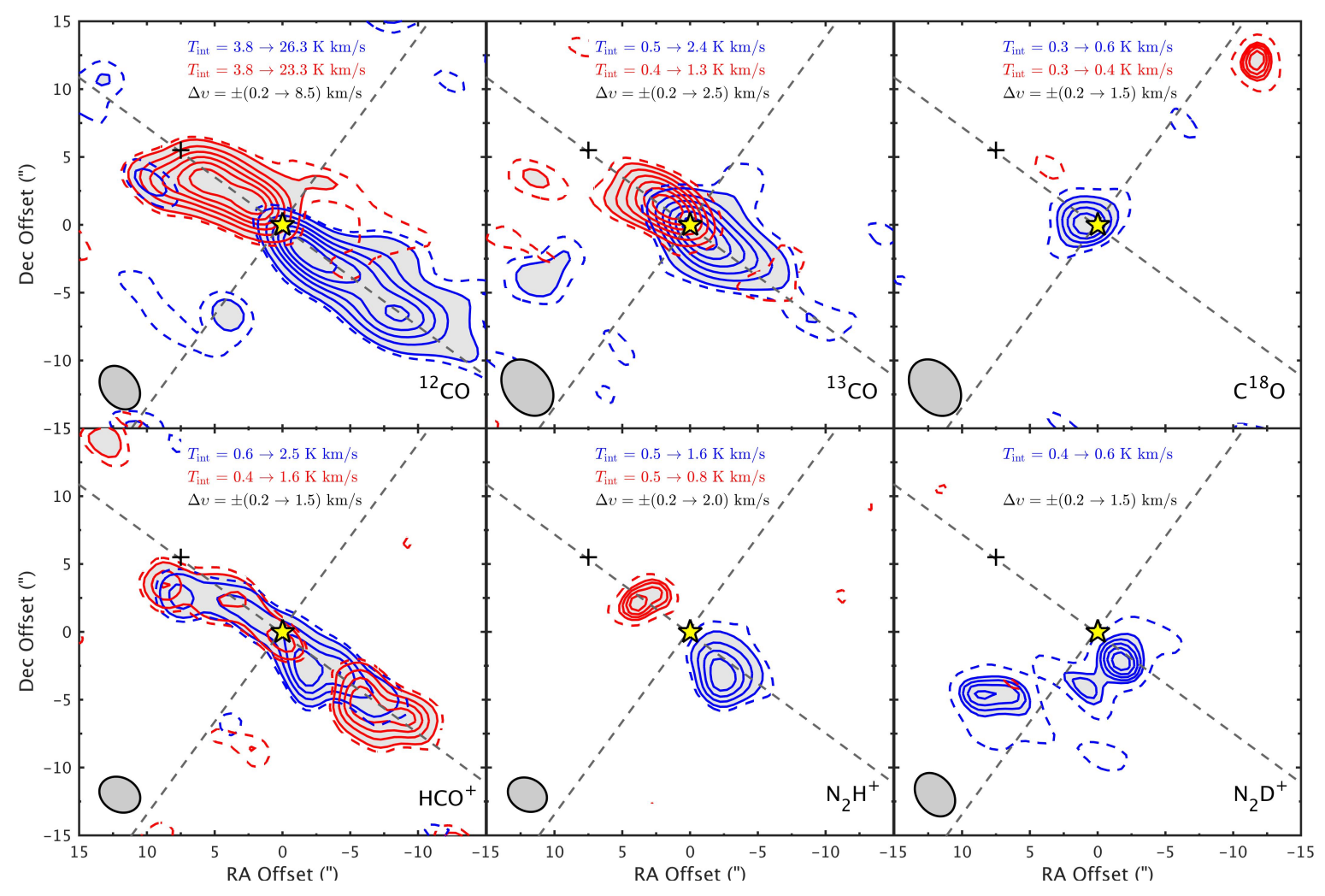

Fig. 2. Integrated emission in the red and blue outflow lobes. The dashed lines indicate the $2 \sigma$ levels and the first thick line indicates the $3 \sigma$ levels. In each panel, the value for the first and last contour in the shaded region are given, together with the velocity intervals over which the emission has been integrated. The beam size and molecules are shown in the lower corners of each panel. Dashed grey lines indicate the cut for the positionvelocity diagram presented in Fig. 4. The location of the central source is indicated with a yellow star and the location of the outflow position discussed in Sect. 4.2.2 is indicated with a plus sign. The maps are centred at $\alpha_{2000}=+15^{\mathrm{h}} 43^{\mathrm{m}} 02 \mathrm{~s} .2 ; \delta_{2000}=-34^{\circ} 09^{\prime} 06.7^{\prime \prime}$.

flow is consistent with recent ALMA observations presented in Jørgensen et al. (2013) and Oya et al. (2014). In those observations, the outflow cavities are clearly detected in the emission from $\mathrm{C}_{2} \mathrm{H}$ (Jørgensen et al. 2013, their Fig. 1), suggesting the presence of a wide-angle wind (see e.g. Lee et al. 2000). The spatial resolution of the $\mathrm{CO}(2-1)$ observations presented here $\left(\sim 3^{\prime \prime}\right)$ is, however, too poor to reveal such variations in the emission on small spatial scales. Consequently, the absence of $\mathrm{HCO}^{+}$emission towards the central source (Jørgensen et al. 2013) is not evident from this dataset either, since the beam size is a factor of $\sim 10$ larger than in the ALMA observations. Also, the optical thickness is expected to be higher in the data presented here. The $\mathrm{HCO}^{+}$peak positions in the outflows are fairly well correlated (within a few arc seconds) with the positions of the ${ }^{12} \mathrm{CO}$ emission maxima at high velocity (see Sect. 4.1 ). The spatial resolution of the dataset does not allow us to tell whether the small spatial differences between $\mathrm{CO}$ and $\mathrm{HCO}^{+}$are due to chemistry or not. One could envision a scenario where $\mathrm{HCO}^{+}$ is partially destroyed in shocked spots along the outflow (Podio et al. 2014). The presence of red-shifted and blue-shifted emission in both outflow lobes could suggest that the $\mathrm{HCO}^{+}$emission stems from the cavity walls (cf. Tappe et al. 2012) where the velocities perpendicular to the outflow axis are expected to be largest. This origin, combined with the fact that the outflow only has a small inclination with respect to the plane of the sky $\left(i=20^{\circ}\right.$, see Sect. 5.1 and Oya et al. 2014), can explain the observed emission.

The $\mathrm{N}_{2} \mathrm{H}^{+}$emission traces the outflow with red-shifted emission in the northeastern lobe and blue-shifted in the southwestern lobe. No emission is detected towards the protostar and the peak positions are located where the ${ }^{12} \mathrm{CO}$ emission peaks at higher velocities (see Fig. 3). At present, we cannot with certainty determine the origin of the $\mathrm{N}_{2} \mathrm{H}^{+}$emission. $\mathrm{N}_{2} \mathrm{H}^{+}$is not an obvious shock tracer and it has only very recently been observed in shocked regions (i.e. L1157-B1, Codella et al. 2013; Podio et al. 2014). The scenario, where $\mathrm{N}_{2} \mathrm{H}^{+}$is not detected towards the protostar is morphologically reminiscent to the $\mathrm{L} 483$ case, where the abundance of $\mathrm{N}_{2} \mathrm{H}^{+}$in the dense central region is reduced as a result of reactions with gas-phase $\mathrm{CO}$ (Jørgensen 2004; Jørgensen et al. 2004). Also in the maps presented in this paper, the $\mathrm{C}^{18} \mathrm{O}$ emission peaks close to the protostar whilst the $\mathrm{N}_{2} \mathrm{H}^{+}$emission peaks in the outflow component where the $\mathrm{CO}$ column density is expected to be lower.

$\mathrm{N}_{2} \mathrm{D}^{+}$is detected in ridge to the south-southeast from IRAS 15398-3359 and only at blue-shifted velocities. The spatial distribution is not well correlated with the extent of the outflow or the protostellar envelope. We can therefore not exclude the possibility that this gas instead is associated with the gas surrounding IRAS 15398-3359.

\section{Analysis}

\subsection{Observed line profiles}

The observed line shapes give important clues when it comes to the relative contribution to the emission from different components. The outflow morphology is clearly evident in the emission from IRAS 15398-3359. However, the emission at velocities within a few $\mathrm{km} \mathrm{s}^{-1}$ from $v_{\text {sys }}$ could also, in addition to the outflow component, have a contribution from the protostellar 


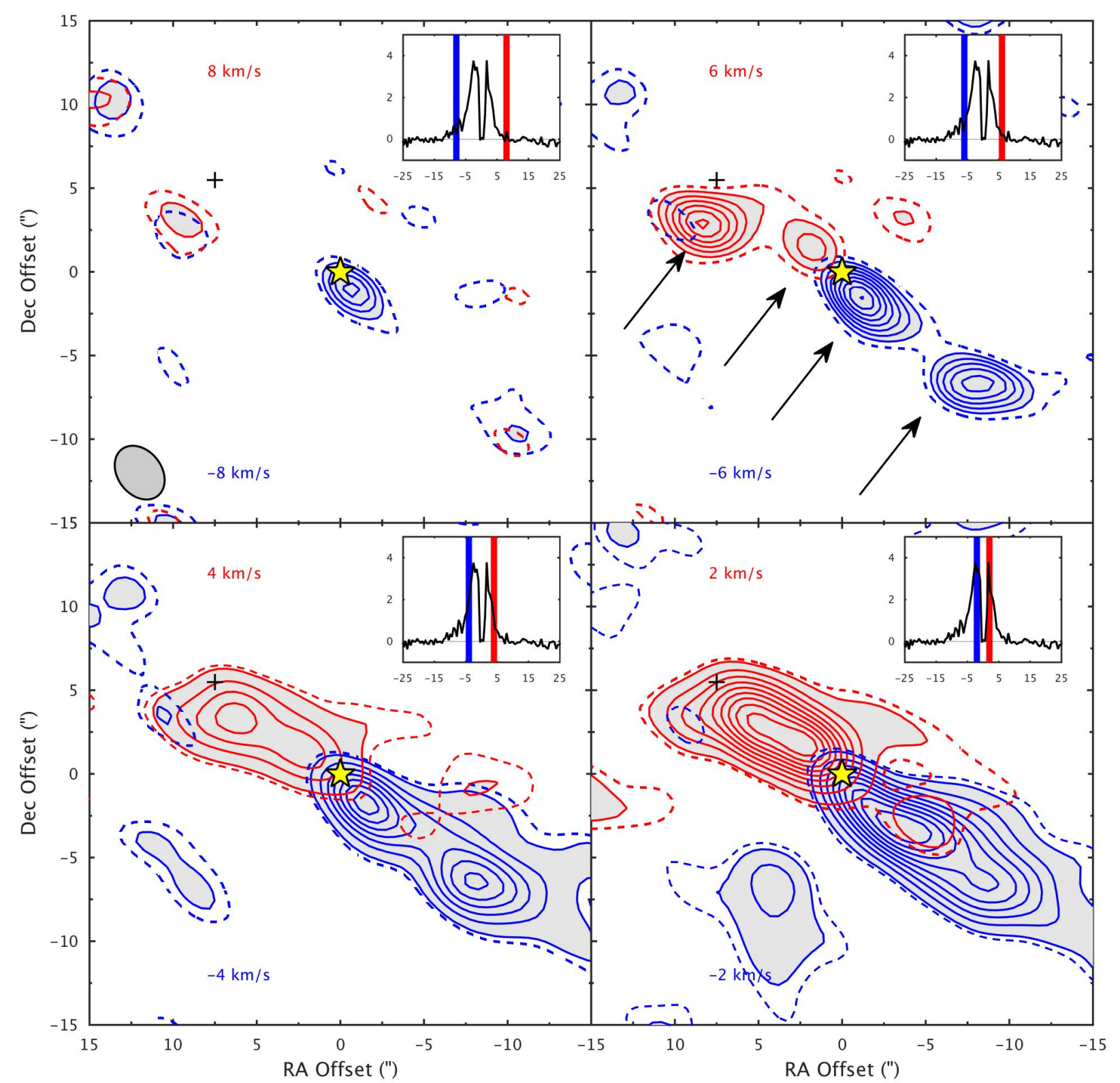

Fig. 3. Integrated ${ }^{12} \mathrm{CO}$ emission in different velocity intervals, where $\Delta v=2 \mathrm{~km} \mathrm{~s}^{-1}$ (inset in each panel). The centre velocities $\left(v-v_{\mathrm{sys}}\right)$ for each bins are indicated in the respective panels. High-velocity gas $\left(\Delta v>5 \mathrm{~km} \mathrm{~s}^{-1}\right)$ is detected in four different spots along the outflow axis (indicated with black arrows in the upper right panel). First solid thick contour is at $3 \sigma$ and the $2 \sigma$ level is indicated with a dashed line.

envelope and/or the protoplanetary disk. Not to forget, the surrounding cloud can have significant impact at the lowest velocities. Inspection of the line profiles in ${ }^{12} \mathrm{CO}$ shows a prominent absorption feature at the systemic velocity. There could be several reasons for this absorption feature. An infalling envelope can give rise to a line profile where the blue-shifted wing component is enhanced with respect to the red-shifted wing component. A search for infall signatures in the emission from $\mathrm{H}_{2} \mathrm{CO}$ and CS was presented in Mardones et al. (1997). This study does not reveal any detectable inflow of gas, however, in a more recent study by Kristensen et al. (2012), the $\mathrm{H}_{2} \mathrm{O}$ line profiles observed with Herschel-HIFI clearly show infall signatures. The $\mathrm{H}_{2} \mathrm{O}$ line profiles were further analysed in a study by Mottram et al. (2013), where the data is consistent with an infall rate of $\sim 3 \times 10^{-5} M_{\odot} \mathrm{yr}^{-1}$. In the IRAS 15398-3359 case, the central absorption could also be affected by the interferometer filtering out the emission on large scales ( $>20^{\prime \prime}$, see Sect. 2$)$, i.e. the low velocity emission. Arguing against this is the smooth distribution of the gas indicating that we also recover most of the emission at low velocities with respect to $v_{\text {sys }}$ (cf. the clumpy distribution in the channel maps presented by Arce et al. 2013). Even though the lack of single-dish data prevents us from putting quantitative numbers on the effect of loss of short spacings, we still find it unlikely that this will significantly affect the emission in the line wings. This emission is believed to originate in the compact regions, either where the jet impact the surrounding medium and/or in the cavity walls of the flow/bow-shock.

\subsection{Radiative transfer modelling}

To investigate to which extent each component contributes to the observed ${ }^{12} \mathrm{CO}(2-1)$ emission lines at various velocities, 

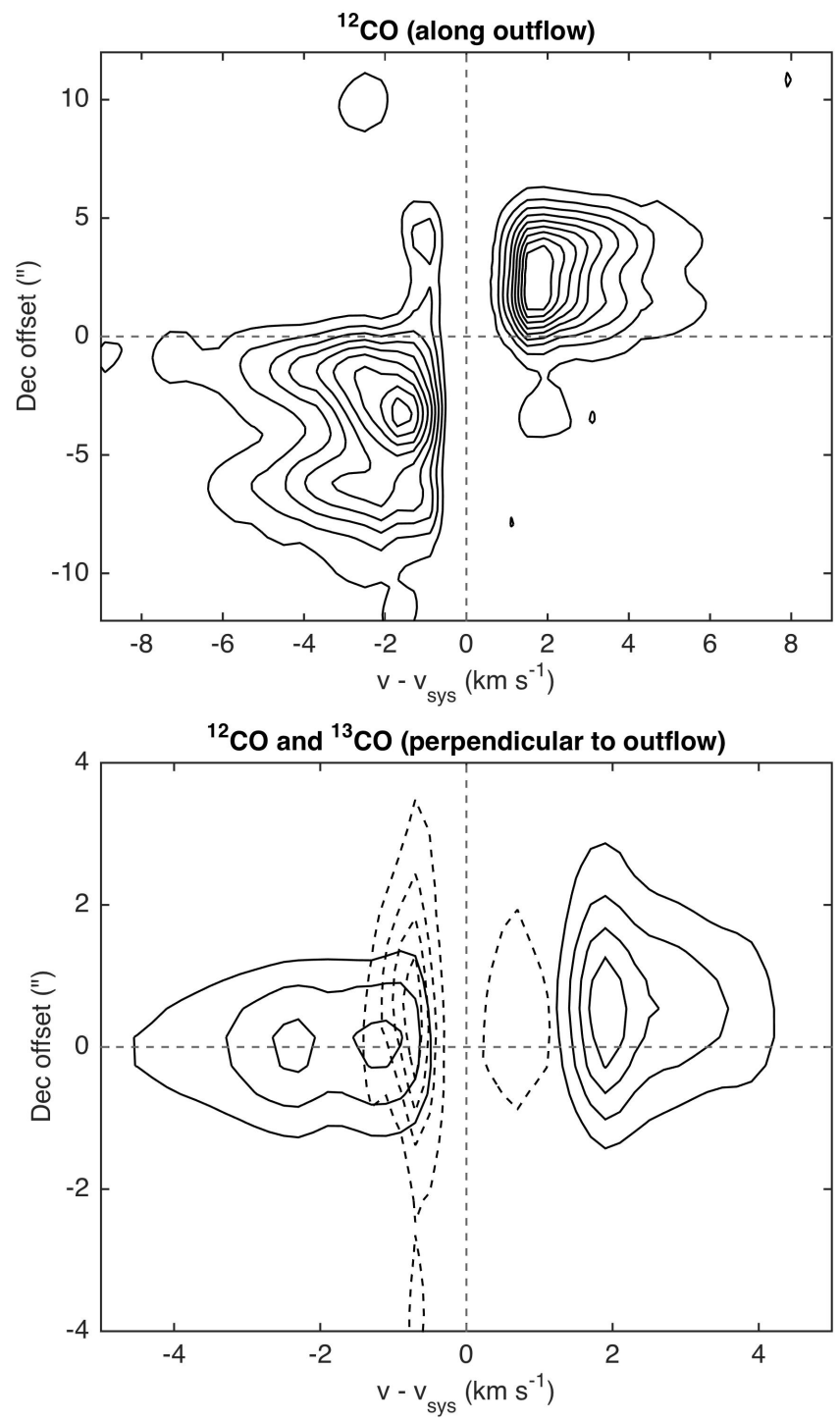

Fig. 4. Position velocity diagram along the outflow direction (upper panel) and along the direction of the presumed disk structure (lower panel). Solid contours are for ${ }^{12} \mathrm{CO}$ and dashed contours are for ${ }^{13} \mathrm{CO}$. The cut along the outflow direction reveals episodic ejection events and weak signs of acceleration along the outflow axis. $v-v_{\text {sys }}$ and the position of the source are indicated with dashed grey lines. Note that the ordinate shows the offset in declination from the central source.

we construct a 3D model of IRAS 15398-3359 via the Line Modelling Engine (LIME; Brinch \& Hogerheijde 2010). This Monte-Carlo radiative transfer code does not put any constraints when it comes to the complexity of the models. The density, temperature, abundance, and velocity structures in the different components are given as analytical descriptions to the LIME code. The irregular Delaunay grid used by LIME is generated by random sampling of the input model. However, the sampling probability is weighted by the density and temperature structure of the model, resulting in a finer grid in the regions with the strongest emission. Once the grid is calculated, LIME starts the iterative process to calculate the rotational level populations. The $\mathrm{CO}$ data file that is used was downloaded from the LAMDA ${ }^{2}$ database (Schöier et al. 2005), where the collisional rate coefficients are taken from Yang et al. (2010). The ortho-to-para ratio for $\mathrm{H}_{2}$ is assumed to take the thermalised value, 3 . When convergence

\footnotetext{
2 http://home.strw.leidenuniv.nl/ moldata/
}

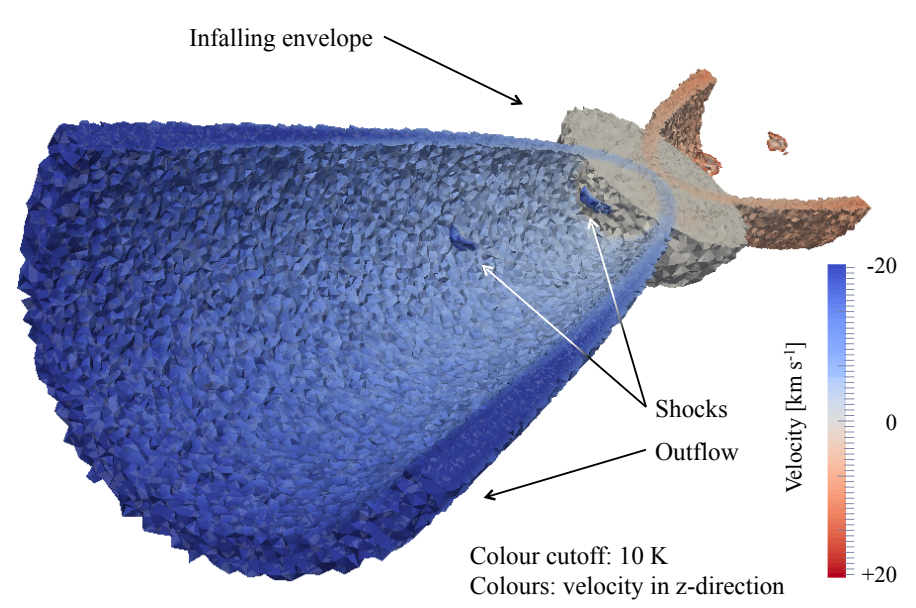

Fig. 5. A cut through the radiative transfer model described in the text (partially transparent). The model is viewed from the south-west direction for clarity. Included in the figure, is the molecular outflow, shocked regions along the jet axis and infalling envelope. Colours show the velocity field in the outflow direction in the regions where the temperature is higher than $10 \mathrm{~K}$. The surrounding cloud component, which is also included in the modelling, is not presented in this figure.

is reached, the model is ray-traced to produce fits files that can be compared to the observations. The image resolution for these fits files is set to $0.25^{\prime \prime}$, which is equivalent to $40 \mathrm{AU}$ at the distance of IRAS 15398-3359. The detailed geometry of the model is discussed in the sections below.

\subsubsection{The model components}

Included in the model are the components that have been proposed to be the major origins of the observed emission from protostellar regions, i.e. infalling envelope, surrounding cloud, outflow cavity, and spot shocks originating in the jet. Although the possibility of a circumstellar disk exist, we do not include this component in the model since these observations are not sensitive to the emission at these spatial scales. A rotationally supported disk is hinted by the $\mathrm{H}_{2} \mathrm{CO}$ emission observed with ALMA (Oya et al. 2014). However, the radius of this feature is likely smaller than $\sim 200 \mathrm{AU}$, i.e. less than the angular resolution obtained here, viz. 400 AU. It should also be mentioned that no velocity gradient perpendicular to the jet direction is detected in the dataset presented in this paper.

The structure of the infalling envelope is based on the bestfit model presented in Mottram et al. (2013) and has a radius, $R_{\text {env }}=4900 \mathrm{AU}$. The velocity field follows a power law,

$v=v_{0}\left(\frac{r}{r_{v_{0}}}\right)^{-p_{v}}$,

where the exponent $p_{v}$ is set to 0.5 and $r_{v_{0}}$ is set to $1000 \mathrm{AU}$. Furthermore, the density profile is described by

$n=n_{0}\left(\frac{r}{r_{n_{0}}}\right)^{-p_{n}}$

where the exponent $p_{n}$ is set to $1.4, r_{n_{0}}$ is set to $6.1 \mathrm{AU}$, and $n_{0}$ is set to $2 \times 10^{9} \mathrm{~cm}^{-3}$. These values are taken from Mottram et al. (2013). However, in this case, the velocity $v_{0}$ at $1000 \mathrm{AU}$ is set to a slightly lower value, viz. $0.1 \mathrm{~km} \mathrm{~s}^{-1}$. Although a higher infall velocity provide a better fit to the width of the line, the larger 


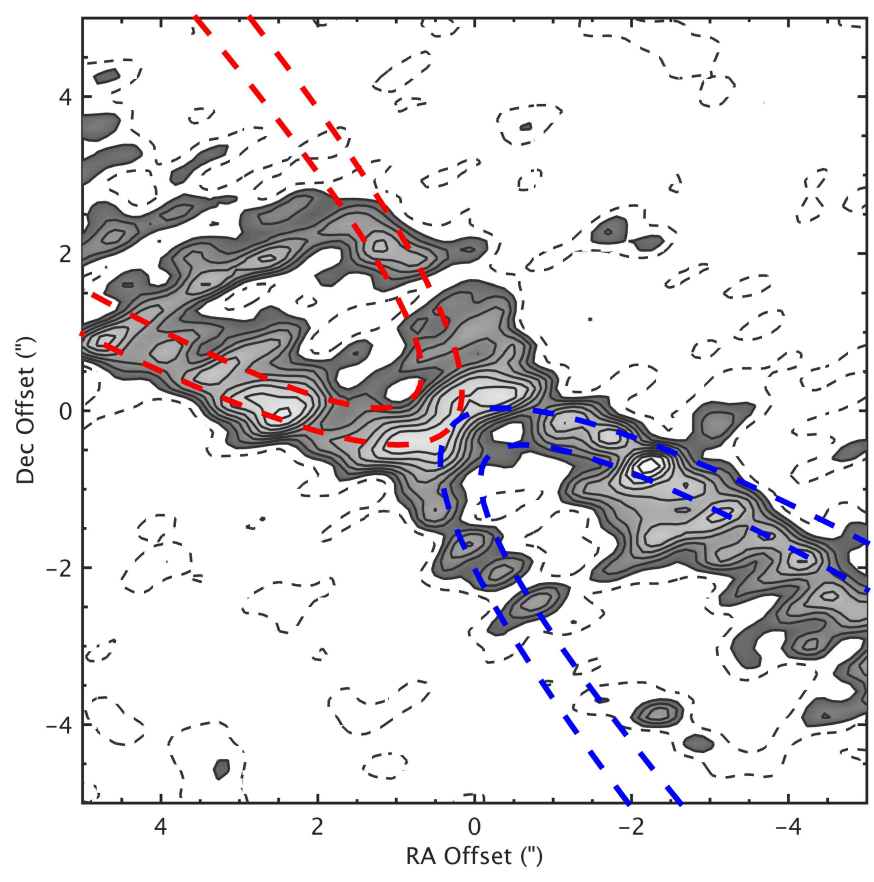

Fig. 6. The model of the outflow cavity (red and blue dashed lines) over plotted on the $\mathrm{C}_{2} \mathrm{H}$ emission acquired with ALMA. The coloured region is where the signal is stronger than $3 \sigma$ and the $1 \sigma$ level is indicated with dashed lines.

gradient also decreases the optical depth in the line, leading to significantly stronger emission than what is observed. Similar to the velocity and density structures, the temperature at $175 \mathrm{AU}$ is set to $30 \mathrm{~K}$ and follows a power law with an exponent of 1.5 (Jørgensen et al. 2013).

The outflow is described by a wind-driven shell (Lee et al. 2000, their Fig. 21) and has an inclination angle of $i=20^{\circ}$ (Oya et al. 2014) with respect to the plane of the sky. It is radially expanding with a Hubble-law velocity structure, and in cylindrical coordinates, the structure and velocity of this shell, is described by

$z=C R^{2}, v_{R}=v_{0} R, v_{z}=v_{0} z$

where $z$ is the distance in the direction of the outflow, $R$ is the radial extent perpendicular to the outflow axis, $v_{R}$ is the velocity in the radial direction, and $v_{z}$ is the velocity in the direction of the outflow axis. The free parameters $v_{0}$ and $C$ are set to $1 \mathrm{~km} \mathrm{~s}^{-1} \operatorname{arcsec}^{-1}$ and $1 \operatorname{arcsec}^{-1}$ to fit the observed velocity extent and geometry of the outflow. The choice of geometry is supported by the PV diagram of $\mathrm{H}_{2} \mathrm{CO}\left(5_{15}-4_{14}\right)$ where an elliptic structure and expansion is obvious (Oya et al. 2014). The thickness of the shell is taken from the observed extent of the $\mathrm{C}_{2} \mathrm{H}$ emission and is set to $100 \mathrm{AU}$ (see Fig. 6). The velocity increases from the inner to the outer region and is highest at the centre of the shell and drops to half at the edges. The length of the blue- and red-shifted outflow cones $\left(L_{\text {blue }}\right.$ and $\left.L_{\text {red }}\right)$ are 2350 and $1400 \mathrm{AU}$, respectively.

In addition to this, bow shocks are added to the inner region of the outflow. The shape of the shocks in cylindrical coordinates follows $z \propto R^{2.4}$ and the velocity decrease with increasing distance from the bow apex (Lee et al. 2000). The distances to the bow shocks are identical to the distances to the peak positions observed in the $\mathrm{CO}$ emission at higher velocities (see upper right panel of Fig. 3). The separation between each shocked region is $800 \mathrm{AU}$ with the first two located $600 \mathrm{AU}$ away from the central source.
The surrounding cloud component is represented by a spherical shell surrounding the entire model. This shell has a thickness, $l_{\text {shell }}=5000 \mathrm{AU}$, and the temperature is set to $10 \mathrm{~K}$. A visualisation of the model is presented in Fig. 5.

\subsubsection{Contribution to the emission line profiles from different components}

Given the large number of free parameters and considerable amount of CPU time needed, a full chi-square analysis is impracticable. Also, such a study would be of little interest because of the many parameters that can be varied. Models that successfully reproduce the observed line profiles, such as that presented in this section should always be considered as one possible solution to the problem. The aim of this study is, therefore, not to reproduce the exact shape of the line profiles in every position of the map, but instead to understand the contribution from each component when taking the most prominent spectral features into account. With our method, we calculate the radiative transfer for a set of models where the contribution from each component is varied between each run. For the outflow component, the velocity field $(v)$, the temperature $(T)$, and density $\left(n_{\mathrm{H}_{2}}\right)$, are varied in a step-by-step manner. The same approach is used for the shocked regions and the surrounding cloud. The $\mathrm{CO}$ abundance is set to $1 \times 10^{-4}$ in all components in the model (see e.g. van Dishoeck \& Black 1987). For each model, 250000 grid points are used and the number of iterations is set to 16 to ensure convergence of the level populations. The synthetic images of the ${ }^{12} \mathrm{CO}$ emission, are sampled with the observed visibilities, inverted, cleaned and finally restored, using Miriad. We thus treat the LIME generated fits cubes in the same way as the observed data. This gives us the short-spacing filtered images that can be directly compared to the observations. In Fig. 7, we present the four different models (M1 to M4) that fit the observations best, when allowing different components (envelope, outflow, shocks, surrounding cloud) to contribute to the emission. The spectrum towards the central position and one outflow position $\left(\Delta \mathrm{RA}=+7.5^{\prime \prime}, \Delta \mathrm{Dec}=+5.5^{\prime \prime}\right)$, as well as a map of the emission (for each individual model) in the blueand red-shifted velocity ranges are presented in this figure. The physical input parameters are summarised in Table 2. In the first model (M1), a pure infall case is considered and no outflow component is present. It is clear that this type of model cannot fully explain the observed emission line profiles. Apart from the obvious fact that no emission is present in the offset position, the line wing strength also decreases with increasing distance from the central source. This is in sharp contrast to the fact that the line wing profiles do not change significantly with increasing distance from the central source.

On the other hand, a pure outflow scenario (where no infall of gas is present) cannot explain the observed line profiles either. This scenario is presented in M2, and in this case, the emission at the systemic velocity is significant compared to the observations, where the strong absorption is clear. In addition, the shape of the lines towards the central region does not resemble the observed lines. We have not been able to reproduce the observed line profiles by adding a cloud component that is filtered out by the interferometer. This is, however, of little interest since the pure outflow scenario is not very likely.

A combination of the first two scenarios can satisfactorily explain the observed emission on both small and large scales (M3). The reason for the strong central reversal of the line profile is in this case the optically thick infalling gas in the central region, absorbing the emission at these frequencies. This model also 
M1 (envelope)
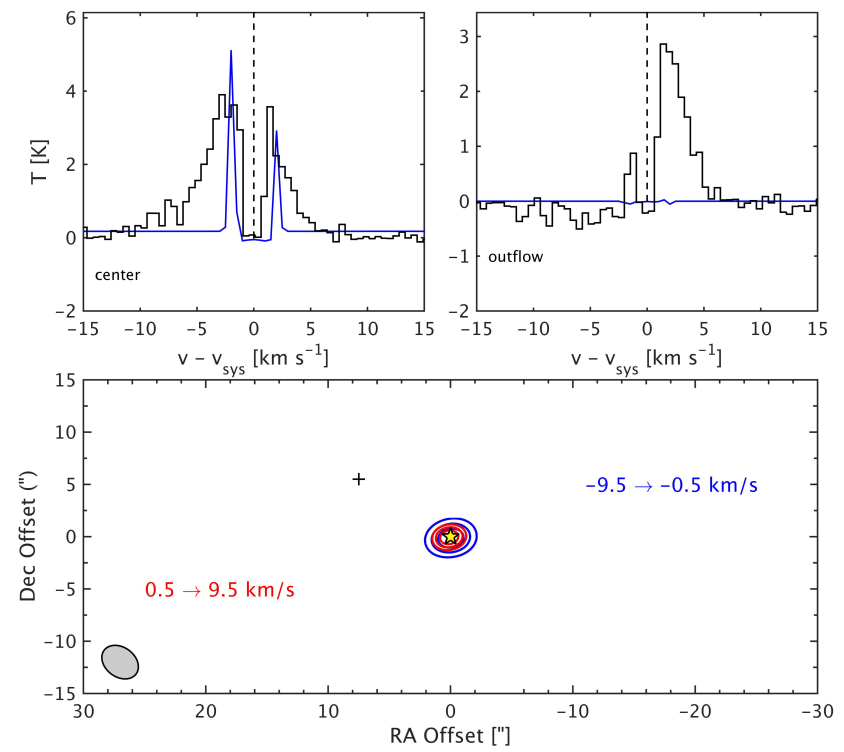

M3 (outflow, envelope \& cloud)
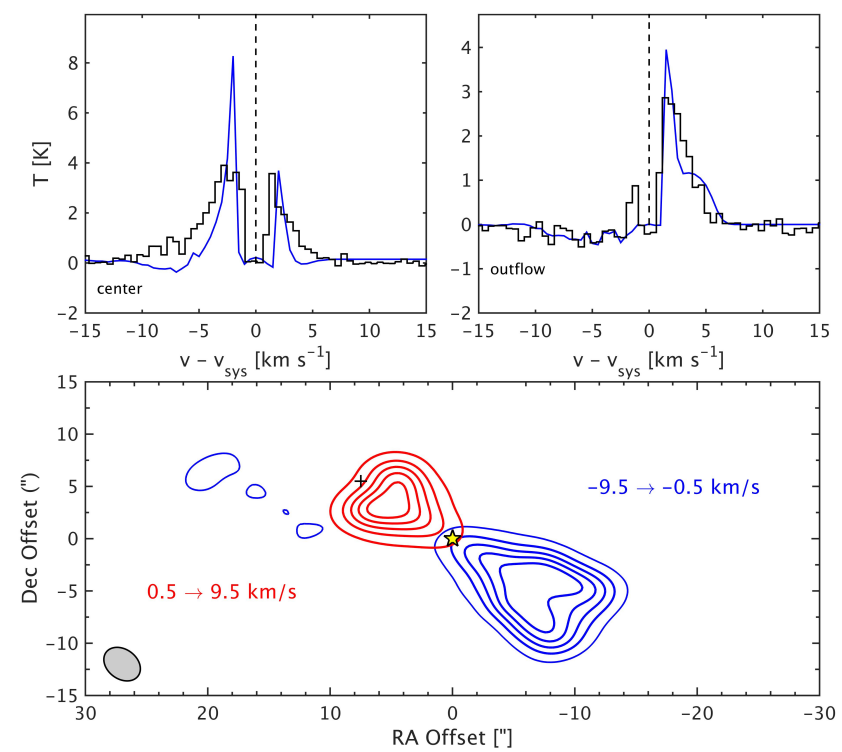

M2 (outflow)
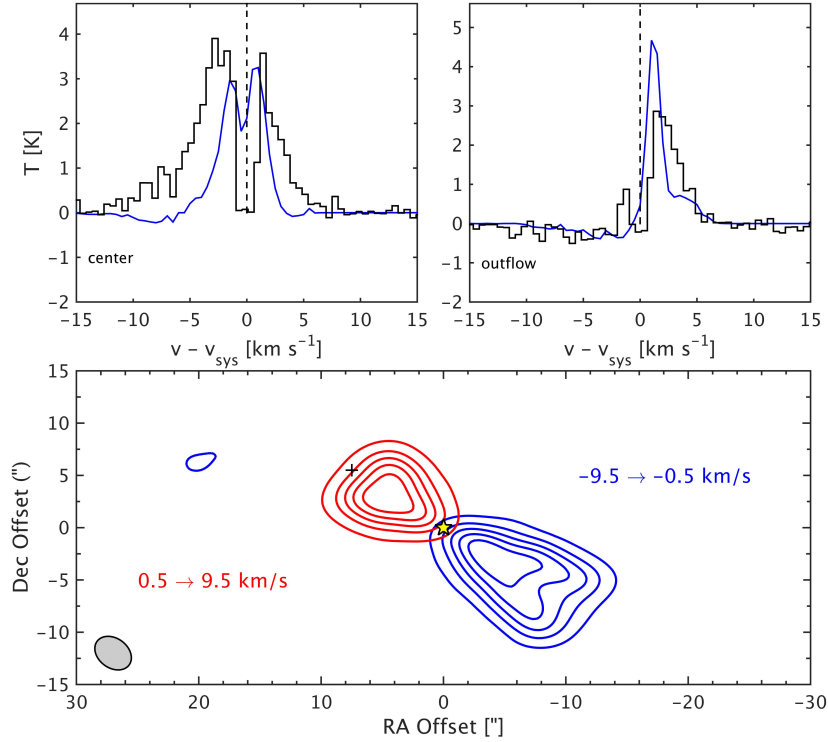

M4 (outflow, envelope \& shocks)
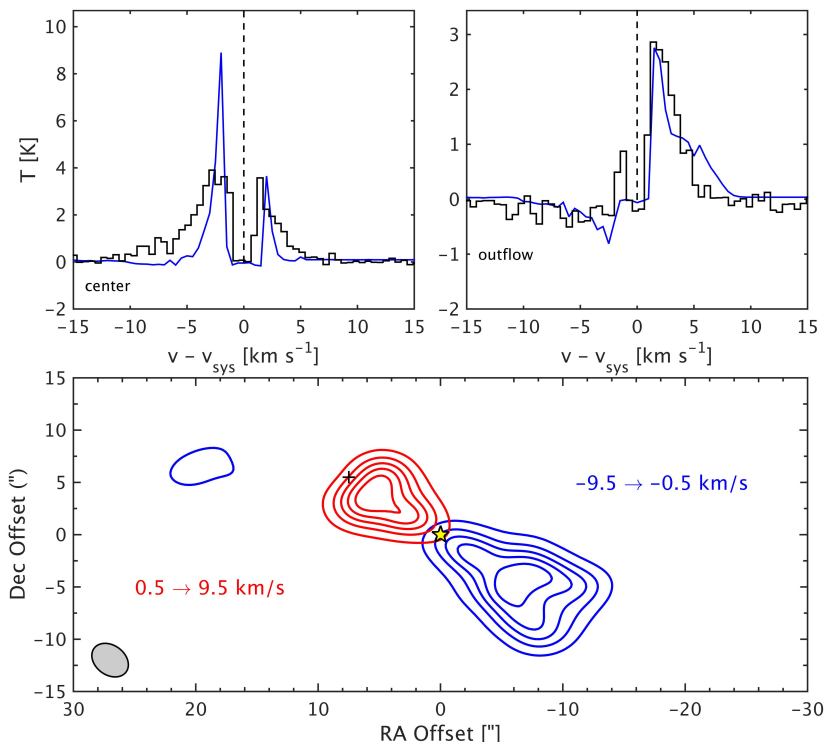

Fig. 7. Four different LIME models discussed in the text (M1 in the upper left corner and M4 in the lower right). In each panel, the spectrum towards the central region and one outflow position is presented. The observed spectra are plotted as black histograms while the modelled spectra are plotted with blue continuous lines. A contour plot is also presented for each model, where the integrated intensity has been calculated over the red and blue line wings, respectively. The beam size is indicated with a grey ellipse.

includes a surrounding cloud, where the $\mathrm{H}_{2}$ column density is $1 \times 10^{20} \mathrm{~cm}^{-2}$. It is clear, however, that this component makes only a small contribution to the line profiles, as is also evident when comparing model M3 with M4 later on. This is not surprising as a result of the exponentially increasing densities and temperatures towards the inner part of the envelope, which is expected to cause a strong absorption feature close to the systemic velocity. This absorption conceals the contribution from a large-scale surrounding cloud that is filtered out by the interferometer. To summarise, even if an extended component is added to the model, the central absorption will still be there owing to the presence of an infalling envelope.

It should be noted here that the assumed velocity profile has a strong impact on the shape of the line profiles. The spatial resolution does not allow us to make a detailed analysis on the variation of the velocity of the gas with distance from the central source at the smallest scales (a few arc-seconds) and the data presented here does not show a clear trend that the velocity is increasing with increasing distance from the central source (Fig. 3). As previously mentioned, however, a pure Hubble-law velocity profile is favoured from the observations of $\mathrm{H}_{2} \mathrm{CO}(\mathrm{Oya}$ et al. 2014) and for that reason we assume that this is the case.

Despite the fact that no obvious bow-shaped structures are present and that no emission at very high velocities is observed in this outflow, we also consider the case where spot shocks along the jet direction contribute to the observed emission line profiles. This case is included for completeness but is not likely based on the very high densities and temperatures that 
Table 2. Parameters in the models discussed in the text and presented in Fig. 7.

\begin{tabular}{ll}
\hline \hline Outflow parameters: & \\
Inclination angle $(i):$ & $20^{\circ}$ \\
Temperature $(T):$ & $100 \mathrm{~K}$ \\
Structure parameter of the shell $(C):$ & $1.0 \mathrm{arcsec}^{-1}$ \\
Velocity parameter of the shell $\left(v_{0}\right):$ & $1.1 \mathrm{~km} \mathrm{~s}^{-1} \mathrm{arcsec}^{-1}$ \\
Length of lobe $\left(L_{\text {blue }}\right):$ & $2350 \mathrm{AU}$ \\
Length of lobe $\left(L_{\mathrm{red}}\right):$ & $1400 \mathrm{AU}$ \\
Envelope parameters: & \\
Radius of envelope $\left(R_{\mathrm{env}}\right):$ & $4900 \mathrm{AU}$ \\
Velocity at $r_{v_{0}}\left(v_{0}\right):$ & $0.1 \mathrm{~km} \mathrm{~s}^{-1}$ \\
Radius where, $v=v_{0}\left(r_{v_{0}}\right):$ & $1000 \mathrm{AU}$ \\
Velocity exponent $\left(p_{v}\right):$ & 0.5 \\
$\mathrm{H}_{2}$ density at $r_{n_{0}}\left(n_{0}\right):$ & $2 \times 10^{9} \mathrm{~cm}^{-3}$ \\
Radius where, $n=n_{0}\left(r_{n_{0}}\right):$ & $6.1 \mathrm{AU}$ \\
Density exponent $\left(p_{n}\right):$ & 1.4 \\
Temperature at $r_{T_{0}}\left(T_{0}\right):$ & $30 \mathrm{~K}$ \\
Radius where, $T=T_{0}\left(R_{T_{0}}\right):$ & $175 \mathrm{AU}$ \\
Temperature exponent $\left(p_{T}\right):$ & 1.5 \\
Shock parameters: & \\
Maximum velocity $\left(v_{\text {max }}\right):$ & $20 \mathrm{~km} \mathrm{~s}^{-1}$ \\
Temperature $(T):$ & $1000 \mathrm{~K}$ \\
H density $\left(n_{\mathrm{H}_{2}}\right):$ & $1 \times 10^{8} \mathrm{~cm}^{-3}$ \\
Shock distance from source $\left(d_{\text {shock }}\right):$ & $600 \mathrm{and} 1400 \mathrm{AU}$ \\
Surrounding cloud parameters: & \\
$\mathrm{H}_{2}$ column density $\left(N_{\mathrm{H}_{2}}\right):$ & $1 \times 10^{20} \mathrm{~cm}^{-2}$ \\
Temperature $(T):$ & $10 \mathrm{~K}$ \\
Shell thickness $\left(l_{\text {shell }}\right):$ & $5000 \mathrm{AU}$ \\
\hline
\end{tabular}

are needed in the relatively confined regions, where shocks can be expected to be present. Although we cannot exclude the contribution from shocked regions completely, this component cannot make a large contribution to the observed emission in this particular source. In model M4, we consider a scenario where an outflow, an infalling envelope and shocked regions along the jet axis are included. The maximum velocity, at the apex of the bow shocks is in the presented model set to, $v_{\max }=20 \mathrm{~km} \mathrm{~s}^{-1}$. Even though the densities and temperatures in the bow shocks are very high $\left(1 \times 10^{8} \mathrm{~cm}^{-3}\right.$ and $1000 \mathrm{~K}$, respectively), the contribution to the line profiles is still moderate and it mostly has an effect at large distances from the central source. In M4, we have also excluded the cloud component to ease the comparison between the different models.

To conclude, the M3 scenario can account for the main features in the line profiles both towards the central region and at offset positions in the outflow component. In addition, this model also reproduces the overall morphology of the $\mathrm{CO}$ emission.

\section{Discussion}

\subsection{A very young outflow source traced by the ${ }^{12} \mathrm{CO}$ emission?}

The outflow from IRAS 15398-3359 was mapped in CO(3-2) using APEX (van Kempen et al. 2009b). From the morphology of the red-shifted and blue-shifted outflow lobes, these authors concluded that the flow had a pole on geometry where the inclination angle with respect to the line of sight was small. The cause of this overlap was, however, likely the large beam size of the APEX telescope at these wavelengths. Inspection of the best-fit model (M3), presented in Sect. 4.2, convolved with the beam-size of APEX, reveals a similar CO(3-2) morphology, as was presented by these authors (their Fig. 13). To conclude, an outflow of short extent appears to be pole-on, when observed with a large beam.

Recent observations with ALMA by Jørgensen et al. (2013) and Oya et al. (2014) revealed two clearly separated outflow lobes suggesting an inclination angle of $20^{\circ}$ with respect to the plane of the sky (Oya et al. 2014), i.e. almost edge-on. From Figs. 2 and 3, it is evident that the emission is confined to a region very close to the central source and that has an edge-on geometry. The absence of emission on large distances from IRAS 15398-3359 $\left(>15^{\prime \prime}\right)$, suggests that this outflow is very young. One obvious argument against this claim could be that the cloud is more dilute at larger distances, but inspection of the ${ }^{13} \mathrm{CO}$ map presented in Tachihara et al. (1996) clearly shows that the cloud has a large extent in the east-west directions $\left(\sim 30^{\prime}\right)$. Also, there are no signs of Herbig-Haro objects on scales larger than 20" from the central source (Heyer \& Graham 1989), supporting the interpretation that this outflow is very young. Assuming that the maximum radial velocity of the outflow is equal to the maximum observed velocity with respect to $v_{\text {sys }}$ (i.e. $\sim 8 \mathrm{~km} \mathrm{~s}^{-1}$, which should be considered as a lower limit to the true maximum outflow velocity) and $i=20^{\circ}$, we estimate the dynamical timescale of the flow to be of the order 500 years (see Sect. 5.3). This puts IRAS 15398-3359 in the same regime as other known outflow sources of very young age (see e.g. Richer 1990; Bourke et al. 2005).

\subsection{Episodic mass ejections}

As mentioned briefly already in Sect. 3.2, recent observations with ALMA (Jørgensen et al. 2013) show that $\mathrm{HCO}^{+}$is absent in the region closest to the protostar. These authors observed the isotopologue $\mathrm{H}^{13} \mathrm{CO}^{+}$and suggest that this ion was destroyed by water vapor that was previously present on these scales. Such a scenario can also explain the emission of $\mathrm{CH}_{3} \mathrm{OH}$ on small spatial scales and the extended observed carbon-chain chemistry. An increased water abundance is expected if the protostar underwent a recent burst in luminosity. As speculated by these authors, such a luminosity increase could be caused by a recent increase in the infall rate towards the protostar, yielding increased shock chemistry in the outflow. From inspection of Fig. 3 it is evident that the emission, with a velocity offset of $\sim 6 \mathrm{~km} \mathrm{~s}^{-1}$ with respect to $v_{\text {sys }}$, peaks at four different locations in the outflow lobes (upper right panel). Two knots are visible on each side of the central source and the projected distance between each knot is $\sim 5^{\prime \prime}$. The more or less equal separation between the knots suggests that these features are due to periodic mass ejections, which are likely accompanied by periodic mass accretion events. The dynamical timescale for these knots is $\sim 100$ years, which is consistent with the analysis presented in Jørgensen et al. (2013), where the luminosity outbursts are estimated to occur on a timescale shorter than 100-1000 years. The position velocity cut along the outflow direction (Fig. 4) clearly shows the episodic events and possibly also weak signs of acceleration, e.g. the "Hubble law" of outflows. A velocity cut along the presumed direction of the disk for ${ }^{12} \mathrm{CO}$ and ${ }^{13} \mathrm{CO}$, does not reveal any signs of rotation. Perhaps the reason for this is that the source is too young to have built up any appreciable disk mass on larger distances.

To summarise, the data suggest that the IRAS 15398-3359 source is very young, likely younger than 1000 years. The emission at high velocities also indicates a shift in the outflow direction (see Fig. 3). This could possibly be due to precession of the ejection axis and/or interaction between the outflow and 
the ambient gas. It is also not clear from this dataset whether IRAS 15398-3359 is a single or binary source (e.g. Dunham et al. 2014), which can complicate the picture even further.

\subsection{Physical properties of the outflowing gas}

Since both ${ }^{13} \mathrm{CO}$ and ${ }^{12} \mathrm{CO}$ was mapped, and detected, in the outflowing gas, we can estimate the opacity of the ${ }^{12} \mathrm{CO}(2-1)$ line. In the outflow positions where the ${ }^{13} \mathrm{CO}$ emission peak (see Fig. 2), and at the systemic velocity, the ${ }^{12} \mathrm{CO}$ to ${ }^{13} \mathrm{CO}$ ratio is close to 1 , implying that the medium is optically thick. However, with increasing velocity, this ratio increases drastically. Unfortunately, the signal-to-noise ratio, for the highvelocity emission in the ${ }^{13} \mathrm{CO}$ line is too low to obtain a firm value on the optical depth. Nevertheless, assuming that the line ratio is increasing with increasing velocity, we can give a lower limit to the ${ }^{12} \mathrm{CO}$ to ${ }^{13} \mathrm{CO}$ line ratio for the high-velocity gas. For velocities $2 \mathrm{~km} \mathrm{~s}^{-1}$ offset from $v_{\text {sys }}$, this ratio is higher than 30 in the red-shifted outflow lobe and higher than 20 in the blue shifted outflow lobe. As this is a lower limit in the velocity regime where ${ }^{13} \mathrm{CO}$ is not detected, and since the line ratio increases dramatically with increasing velocity, we find it reasonable to assume that the lines are optically thin in the line wings. For the analysis presented here, we only take this optically thin regime into account. Thus, the mass estimates should be interpreted as lower limits to the true mass in the outflow lobes.

In the optically thin regime, the integrated emission in the line wings can be converted to a column density, assuming LTE conditions and using standard techniques (see e.g. Wilson et al. 2009). Since no other rotational lines of CO have been observed on these small scales, it is not possible to get a robust estimate on the excitation temperature along the outflow. If we assume a typical excitation temperature of $100 \mathrm{~K}$ in the outflow (see e.g. van Kempen et al. 2009a), and a $\mathrm{CO} / \mathrm{H}_{2}$ ratio of $10^{-4}$ (see e.g. van Dishoeck \& Black 1987), the $\mathrm{H}_{2}$ column density is estimated at $3 \times 10^{20} \mathrm{~cm}^{-2}$ in the red-shifted and blue-shifted outflow lobes. Owing to the uncertainty of this value, it is worth noting that the column density does not change by more than a factor of 5 when the excitation temperature is varied between 10 and $500 \mathrm{~K}$. Given the extent $\left(L_{\text {lobe }} \simeq 3700 \mathrm{AU}\right)$ and width ( $\sim 700 \mathrm{AU})$ of the flow, these numbers correspond to a total outflow mass of $3 \times 10^{-4} M_{\odot}$. The inferred value is consistent with the estimates presented by Dunham et al. (2014, $M_{\text {lobe }} \geq$ $\left.4 \times 10^{-4} M_{\odot}\right)$ and Yildiz et al. $\left(2015, M_{\text {lobe }} \simeq 3 \times 10^{-4} M_{\odot}\right)$, and it is small when compared to other Class 0 sources (see e.g. Cabrit \& Bertout 1992; Wu et al. 2004; Curtis et al. 2010; Dunham et al. 2014; Bjerkeli et al. 2013, where estimates typically are higher than $10^{-4} M_{\odot}$ ). The outflow mass is also very small compared to the protostellar mass, which has been estimated at $4 \times 10^{-2} M_{\odot}$ (Oya et al. 2014). This confirms that only a small amount of gas has been ejected out into the outflow and it supports the conclusion that the source is very young.

The other characteristic flow parameters can be estimated from the flow extent, mass, and velocity. First, the dynamical timescale of the flow is inferred from the extent and maximum observed velocity in the red-shifted $\left(v_{\max } \simeq 6 \mathrm{~km} \mathrm{~s}^{-1}\right)$ and blue-shifted $\left(v_{\max } \simeq 8 \mathrm{~km} \mathrm{~s}^{-1}\right)$ outflow lobe, deprojected by the inclination angle with respect to the line of sight $\left(t_{\mathrm{dyn}}=L_{\mathrm{lobe}} \cos (i) / v_{\max }\right)$. The maximum deprojected red-shifted velocity is thus $18 \mathrm{~km} \mathrm{~s}^{-1}$ and the corresponding number for the blue-shifted flow is $23 \mathrm{~km} \mathrm{~s}^{-1}$. This gives a dynamical timescale of the outflow between 400 and 500 years. This is a factor of two lower than the value reported by Yildiz et al. (2015), based on APEX-CHAMP+ observations. When inferring the energetic parameters, we use the method suggested by Downes \& Cabrit (2007). For the momentum, the characteristic velocity, $v_{\text {char }}$ (Lada \& Fich 1996), is used and no inclination correction is applied. To derive the characteristic velocity, we use the method described in André et al. (1990), where the intensityweighted absolute velocity averaged over the mapped area is calculated. The derived flow momentum is $2 \times 10^{-4} M_{\odot} \mathrm{km} \mathrm{s}^{-1}$ and $4 \times 10^{-4} M_{\odot} \mathrm{km} \mathrm{s}^{-1}$, in the red- and blue-shifted outflow lobe, respectively. For the kinetic energy, we use a factor of 5 when correcting for inclination (Downes \& Cabrit 2007, their Figs. 4 and 5), and we arrive at $2 \times 10^{40} \mathrm{erg}$ and $5 \times 10^{40} \mathrm{erg}$ in the red- and blue-shifted lobe, respectively. The force of the outflow (momentum rate) is measured at $\sim 1 \times 10^{-6} M_{\odot} \mathrm{km} \mathrm{s}^{-1} \mathrm{yr}^{-1}$, i.e. slightly lower than the number presented in Yildiz et al. (2015). Compared to the results presented in that paper, the mechanical luminosity is also estimated at a slightly lower value, $\sim 1 \times 10^{-3} L_{\odot}$, which can be compared to the bolometric luminosity of the source, $1.8 L_{\odot}$ (Jørgensen et al. 2013). Unfortunately, the limited spatial resolution of the dataset presented here does not allow us to estimate the infall rate. A meaningful comparison with the mass-loss rate would, however, also be prohibited by the unknown size of the presumed protostellar disk. The mass-loss rate can readily be obtained from the momentum rate and an assumed velocity of the wind (see e.g. Goldsmith et al. 1984). Setting the wind velocity equal to the maximum flow velocity we derive a mass-loss rate, $\sim 7 \times 10^{-8} M_{\odot} \mathrm{yr}^{-1}$. To conclude, the mass-loss rate, the mechanical luminosity, momentum, momentum rate, and kinetic energy are all estimated at relatively low values.

Compared to previous studies of outflows (cf. Wu et al. 2004; Curtis et al. 2010; Dunham et al. 2014), the values presented here fall at the lower end. In those papers, mass-loss rates are typically reported to be higher than $10^{-9} M_{\odot} \mathrm{yr}^{-1}$ and the mechanical luminosity is for the bulk part of observed outflows higher than $10^{-3} L_{\odot}$. The momentum, momentum rates, and kinetic energies are also typically found to be higher than $10^{-3} M_{\odot} \mathrm{km} \mathrm{s}^{-1}$, $10^{-6} M_{\odot} \mathrm{km} \mathrm{s}^{-1} \mathrm{yr}^{-1}$, and $10^{41} \mathrm{erg}$, respectively. It is therefore worth noting again that IRAS $15398-3359$ is a Class 0 source. Younger outflow sources are typically found to be more energetic than their evolved counterparts (see e.g. Bontemps et al. 1996). Why IRAS 15398-3359 shows such characteristics as presented here is not entirely clear to us, but one reason may be that only a small amount of gas and dust up to now has been entrained by the outflow (hinted by the relatively low outflow mass). Another important aspect may be that IRAS 15398-3359 is uncommon, in the sense that it has a highly variable accretion rate. If the protostar for long periods stay in a low accretion rate mode, this will also have an impact on the energetics of the flow.

\section{Conclusions}

We present maps of $\mathrm{CO}, \mathrm{HCO}^{+}, \mathrm{N}_{2} \mathrm{H}^{+}$, and $\mathrm{N}_{2} \mathrm{D}^{+}$towards the IRAS 15398-3359 region observed with the Sub-Millimeter Array (SMA). These data were taken with a higher spatial resolution $\left(2.6^{\prime \prime}-4.6^{\prime \prime}\right)$ than has previously been done for CO. From the analysis of these data and from comparison with recent ALMA observations, we conclude the following:

${ }^{12} \mathrm{CO}(2-1)$ and ${ }^{13} \mathrm{CO}(2-1)$ are detected towards the IRAS 15398-3359 region and are shown to have an outflow origin. $\mathrm{C}^{18} \mathrm{O}(2-1)$ is detected towards the central region and only at blue-shifted velocities. $\mathrm{HCO}^{+}(3-2)$, and $\mathrm{N}_{2} \mathrm{H}^{+}(3-2)$ is also tracing the outflow emission, while $\mathrm{N}_{2} \mathrm{D}^{+}(3-2)$ is absent in the 
Table 3. Physical parameters of the outflow derived from the ${ }^{12} \mathrm{CO}$ emission maps.

\begin{tabular}{lll}
\hline \hline & Red lobe: & Blue lobe: \\
$M_{\text {lobe }}\left(M_{\odot}\right):$ & $2 \times 10^{-4}$ & $2 \times 10^{-4}$ \\
$L_{\text {lobe }}(\mathrm{pc}):$ & $8 \times 10^{-3}$ & $1 \times 10^{-2}$ \\
$R_{\text {lobe }}(\mathrm{pc}):$ & $3 \times 10^{-3}$ & $4 \times 10^{-3}$ \\
$v_{\text {char }}\left(\mathrm{km} \mathrm{s}^{-1}\right):$ & 5.1 & 7.4 \\
$v_{\text {max }}\left(\mathrm{km} \mathrm{s}^{-1}\right):$ & 6.0 & 8.0 \\
$t_{\text {dyn }}(\mathrm{yr}):$ & $4 \times 10^{2}$ & $5 \times 10^{2}$ \\
Momentum $\left(M_{\odot} \mathrm{km} \mathrm{s}^{-1}\right):$ & $2 \times 10^{-4}$ & $4 \times 10^{-4}$ \\
Kinetic energy $\left(\mathrm{erg}^{2}\right)$ & $2 \times 10^{40}$ & $5 \times 10^{40}$ \\
Momentum rate $\left(M_{\odot} \mathrm{km} \mathrm{s}^{-1} \mathrm{yr}^{-1}\right):$ & $4 \times 10^{-7}$ & $1 \times 10^{-6}$ \\
Mechanical luminosity $\left(L_{\odot}\right):$ & $3 \times 10^{-4}$ & $1 \times 10^{-3}$ \\
Wind mass-loss rate $\left(M_{\odot} \mathrm{yr}^{-1}\right):$ & $3 \times 10^{-8}$ & $4 \times 10^{-8}$ \\
\hline
\end{tabular}

outflow and only detected to the southwest. This suggests a non-outflow origin for this particular species. From analysis of the $\mathrm{CO}$ emission, where signs of episodic mass ejections are obvious, we conclude that the IRAS 15398-3359 source is very young, possibly younger than $\sim 1000$ years. The physical properties of the outflow, such as the mass, momentum, momentum rate, mechanical luminosity, kinetic energy, and mass-loss rate are estimated at relatively low values. The data does not reveal any signs of a rotating disk-like structure on these scales. A full $3 \mathrm{D}$ radiative transfer model of the system, using the LIME code, explains the observations well. In order to reproduce the kinematical features in the CO line profiles as well as the morphology of the flow, three different components are needed, viz. a circumstellar envelope, a surrounding cloud, and a wide-angle wind outflow.

Acknowledgements. We thank the anonymous referee for a thorough report that greatly improved the quality of the paper. This research was supported by the Swedish research council (VR) through the contract 637-2013472 to Per Bjerkeli and by the Lundbeck Foundation Junior Group Leader Fellowship to Jes K. Jørgensen. Centre for Star and Planet Formation is funded by the Danish National Research Foundation. We also acknowledge support from National Science Foundation grant 1008800 and EU A-ERC grant 291141 CHEMPLAN. The Submillimeter Array is a joint project between the Smithsonian Astrophysical Observatory and the Academia Sinica Institute of Astronomy and Astrophysics and is funded by the Smithsonian Institution and the Academia Sinica. This paper makes use of the following ALMA data: ADS/JAO.ALMA\#2011.0.00628.S. ALMA is a partnership of ESO (representing its member states), NSF (USA) and NINS (Japan), together with NRC (Canada), NSC and ASIAA (Taiwan), and KASI (Republic of Korea), in cooperation with the Republic of Chile. The Joint ALMA Observatory is operated by ESO, AUI/NRAO and NAOJ.

\section{References}

André, P., Martin-Pintado, J., Despois, D., \& Montmerle, T. 1990, A\&A, 236, 180

André, P., Ward-Thompson, D., \& Barsony, M. 1993, ApJ, 406, 122
Arce, H. G., Mardones, D., Corder, S. A., et al. 2013, ApJ, 774, 39

Banerjee, R., \& Pudritz, R. E. 2006, ApJ, 641, 949

Bjerkeli, P., Liseau, R., Nisini, B., et al. 2013, A\&A, 552, L8

Bontemps, S., Andre, P., Terebey, S., \& Cabrit, S. 1996, A\&A, 311, 858

Bourke, T. L., Crapsi, A., Myers, P. C., et al. 2005, ApJ, 633, L129

Brinch, C., \& Hogerheijde, M. R. 2010, A\&A, 523, A25

Cabrit, S., \& Bertout, C. 1992, A\&A, 261, 274

Chen, X., Arce, H. G., Zhang, Q., et al. 2013, ApJ, 768, 110

Codella, C., Viti, S., Ceccarelli, C., et al. 2013, ApJ, 776, 52

Curtis, E. I., Richer, J. S., Swift, J. J., \& Williams, J. P. 2010, MNRAS, 408, 1516

Downes, T. P., \& Cabrit, S. 2007, A\&A, 471, 873

Dunham, M. M., Arce, H. G., Mardones, D., et al. 2014, ApJ, 783, 29

Goldsmith, P. F., Snell, R. L., Hemeon-Heyer, M., \& Langer, W. D. 1984, ApJ, 286, 599

Heyer, M. H., \& Graham, J. A. 1989, PASP, 101, 816

Ho, P. T. P., Moran, J. M., \& Lo, K. Y. 2004, ApJ, 616, L1

Jørgensen, J. K. 2004, A\&A, 424, 589

Jørgensen, J. K., Schöier, F. L., \& van Dishoeck, E. F. 2004, A\&A, 416, 603

Jørgensen, J. K., Visser, R., Sakai, N., et al. 2013, ApJ, 779, L22

Jørgensen, J. K., Visser, R., Williams, J. P., \& Bergin, E. A. 2015, A\&A, 579, A 23

Kristensen, L. E., van Dishoeck, E. F., Bergin, E. A., et al. 2012, A\&A, 542, A8 Lada, C. J., \& Fich, M. 1996, ApJ, 459, 638

Lee, C.-F., Mundy, L. G., Reipurth, B., Ostriker, E. C., \& Stone, J. M. 2000, ApJ, 542, 925

Lombardi, M., Lada, C. J., \& Alves, J. 2008, A\&A, 489, 143

Machida, M. N., Inutsuka, S.-i., \& Matsumoto, T. 2008, ApJ, 676, 1088

Mardones, D., Myers, P. C., Tafalla, M., et al. 1997, ApJ, 489, 719

Mottram, J. C., van Dishoeck, E. F., Schmalzl, M., et al. 2013, A\&A, 558, A126

Oya, Y., Sakai, N., Sakai, T., et al. 2014, ApJ, 795, 152

Podio, L., Lefloch, B., Ceccarelli, C., Codella, C., \& Bachiller, R. 2014, A\&A, 565, A64

Pudritz, R. E., Ouyed, R., Fendt, C., \& Brandenburg, A. 2007, in Protostars and Planets V, 277

Richer, J. S. 1990, MNRAS, 245, 24

Sakai, N., Sakai, T., Hirota, T., \& Yamamoto, S. 2011, in EAS Pub. Ser. 52, eds. M. Röllig, R. Simon, V. Ossenkopf, \& J. Stutzki, 235

Sault, R. J., Teuben, P. J., \& Wright, M. C. H. 1995, in Astronomical Data Analysis Software and Systems IV, eds. R. A. Shaw, H. E. Payne, \& J. J. E. Hayes, ASP Conf. Ser., 77, 433

Schöier, F. L., van der Tak, F. F. S., van Dishoeck, E. F., \& Black, J. H. 2005, A\&A, 432, 369

Shang, H., Li, Z.-Y., \& Hirano, N. 2007, in Protostars and Planets V, 261

Shirley, Y. L., Evans, II, N. J., Rawlings, J. M. C., \& Gregersen, E. M. 2000, ApJS, 131, 249

Tachihara, K., Dobashi, K., Mizuno, A., Ogawa, H., \& Fukui, Y. 1996, PASJ, 48, 489

Tappe, A., Forbrich, J., Martín, S., Yuan, Y., \& Lada, C. J. 2012, ApJ, 751, 9 van Dishoeck, E. F., \& Black, J. H. 1987, in NATO ASIC Proc. 210: Physical Processes in Interstellar Clouds, eds. G. E. Morfill, \& M. Scholer, 241

van Kempen, T. A., van Dishoeck, E. F., Güsten, R., et al. 2009a, A\&A, 507, 1425

van Kempen, T. A., van Dishoeck, E. F., Hogerheijde, M. R., \& Güsten, R. 2009b, A\&A, 508, 259

Wilner, D. J., \& Welch, W. J. 1994, ApJ, 427, 898

Wilson, T. L., Rohlfs, K., \& Hüttemeister, S. 2009, in Tools of Radio Astronomy (Springer-Verlag), 18

Wu, Y., Wei, Y., Zhao, M., et al. 2004, A\&A, 426, 503

Yang, B., Stancil, P. C., Balakrishnan, N., \& Forrey, R. C. 2010, ApJ, 718, 1062

Yıldız, U. A., Kristensen, L. E., van Dishoeck, E. F., et al. 2015, A\&A, 576, A109 